Residual thermal strain in thick GaN epifilms revealed by cross-sectional Raman scattering and cathodoluminescence spectra

This article has been downloaded from IOPscience. Please scroll down to see the full text article. 2007 Semicond. Sci. Technol. 22896

(http://iopscience.iop.org/0268-1242/22/8/012)

The Table of Contents and more related content is available

Download details:

IP Address: 140.112.113.225

The article was downloaded on 26/06/2009 at 11:04

Please note that terms and conditions apply. 


\title{
Residual thermal strain in thick GaN epifilms revealed by cross-sectional Raman scattering and cathodoluminescence spectra
}

\author{
F C Wang ${ }^{1}$, C L Cheng ${ }^{1}$, Y F Chen ${ }^{1}$, C F Huang ${ }^{2}$ and C C Yang ${ }^{2}$ \\ ${ }^{1}$ Department of Physics, National Taiwan University, Taipei 106, Taiwan \\ ${ }^{2}$ Graduate Institute of Electro-Optical Engineering and Department of Electrical \\ Engineering, National Taiwan University, Taipei 106, Taiwan \\ E-mail: yfchen@phys.ntu.edu.tw
}

Received 9 April 2007, in final form 15 June 2007

Published 12 July 2007

Online at stacks.iop.org/SST/22/896

\begin{abstract}
Strain can significantly alter the physical properties of a solid. We demonstrate that in a thick GaN epilayer there exists a residual thermal strain along the growth direction. This result is clearly revealed by cathodoluminescence spectra, in which the band gap of the GaN film decreases with distance away from the epifilm-substrate interface. This result is further confirmed by Raman scattering spectra in which the phonon modes show a red shift along the growth direction. Our finding is important for the understanding and application of nitride semiconductors.
\end{abstract}

(Some figures in this article are in colour only in the electronic version)

\section{Introduction}

In recent years, GaN-based materials have attracted considerable attention because of their application in the fabrication of optoelectronic devices, including light emitting diodes (LED), laser diodes (LD) and high power and high temperature transistors [1-3]. However, an important obstacle in growing $\mathrm{GaN}$ layers on crystalline substrates, such as sapphire, $\mathrm{SiC}$ or $\mathrm{Si}$, is related to the large lattice mismatch and the difference in the thermal expansion coefficients between the epitaxial layer and the substrate, which would cause large biaxial stresses in the epitaxial layers. For example, it is well known that the stress is compressive in $\mathrm{GaN}$ grown on sapphire, whereas its sign is opposite in GaN deposited on $6 \mathrm{H}-\mathrm{SiC}$ and $\mathrm{Si}$ [4-6]. Consequently, most GaN heteroepitaxial layers inevitably contain a residual stress. It is also known that nitride semiconductors are excellent piezoelectric materials [3]. A small stress can have a significant influence on their physical properties, and therefore on the performance of optoelectronic devices based on nitride semiconductors. However, up to now, the study of the residual stress in nitride semiconductors is rather limited [7].
\end{abstract}

In this paper, we report an investigation by means of crosssectional micro-Raman scattering and cathodoluminescence (CL) spectra to uncover the variation of the residual stress in thick GaN epifilms. Micro-Raman spectroscopy is a useful tool to spatially resolve internal strains and stresses in semiconductor heterostructures through the high resolution in determining phonon frequencies, and it represents an effective and nondestructive technique, which is accessible in most laboratories. Meanwhile, with its sharply focused electron beam, CL spectroscopy can locally probe the optical properties of an epilayer. It offers us a convenient way to determine the variation of stress according to the change of the energy band gap due to residual strains. Therefore, both techniques can provide complementary information to reveal the local variation of residual stress in an epifilm, which is quite unique among all currently available tools.

\section{Experiment}

The undoped $\sim 4.6 \mu \mathrm{m}$ thick hexagonal GaN epifilms studied here were grown by metalorganic chemical vapour deposition (MOCVD) directly on a sapphire (0 0001$)$ substrate. A $25 \mathrm{~nm}$ 


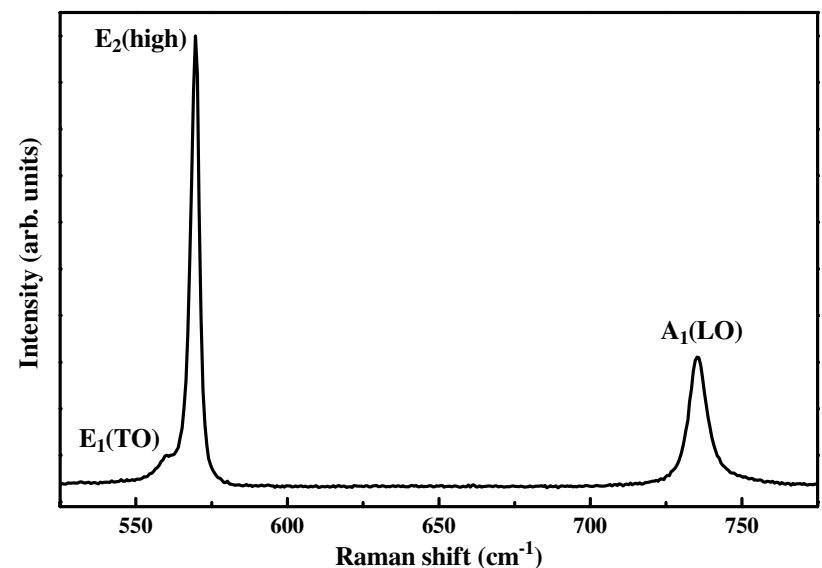

Figure 1. Surface Raman scattering spectrum measured from the GaN epilayer at room temperature.

thick GaN nucleation layer was first deposited prior to the growth of the top $\mathrm{GaN}$ layer at $535{ }^{\circ} \mathrm{C}$. The growth was then performed at $1070{ }^{\circ} \mathrm{C}$. Raman scattering spectra were recorded by a Jobin-Yvon T64000 triple spectrometer equipped with a liquid-nitrogen-cooled charged-coupled device (CCD) detector at room temperature in the backscattering geometry. The excitation laser source with a wavelength of $514.5 \mathrm{~nm}$ at a power of $100 \mathrm{~mW}$ was used and the spectral resolution was less than $0.5 \mathrm{~cm}^{-1}$. The spot size of the laser beam was first measured with a micrometer, which is around $1 \mu \mathrm{m}$, and the power across the light spot approximately forms a Gaussion distribution. CL experiments were performed at room temperature with a JEOL-JSM 6500 scanning electron microscopy (SEM) using a $5 \mathrm{kV}$ electron beam. Excitation spectra were gathered by a photomultiply tube (PMT) detector with a MonoCL3 CL system (Gatan Instruments).

\section{Results and discussion}

Figure 1 shows the Raman scattering spectrum for the laser light incident normal to the surface of the GaN epilayer. Before analysing the Raman spectrum, let us recall that $\mathrm{GaN}$ crystallizes in a hexagonal wurtzite structure with four atoms in the unit cell and belongs to the space group $C_{6 v}^{4}\left(C 6_{3} m c\right)$. Two $A_{1}$, two $E_{1}$, two $E_{2}$ and two $B_{1}$ modes are predicted by the group theory. One $\mathrm{A}_{1}$ and one $\mathrm{E}_{1}$ are acoustic vibrations. The $E_{2}$ modes are Raman active, the $A_{1}$ and the $E_{1}$ modes are both Raman and infrared active, and the $\mathrm{B}_{1}$ modes are silent [8]. The six Raman active phonons-i.e., $\mathrm{A}_{1}(\mathrm{TO}), \mathrm{A}_{1}(\mathrm{LO})$, $\mathrm{E}_{1}(\mathrm{TO}), \mathrm{E}_{1}(\mathrm{LO}), \mathrm{E}_{2}$ (low) and $\mathrm{E}_{2}$ (high) — can be observed for first-order Raman scattering. In figure 1, three Raman active phonon peaks can be clearly observed, including the $\mathrm{E}_{1}(\mathrm{TO})$ at $\sim 559.6 \mathrm{~cm}^{-1}$, the $\mathrm{E}_{2}$ (high) at $\sim 569.6 \mathrm{~cm}^{-1}$ and the $\mathrm{A}_{1}(\mathrm{LO})$ at $\sim 735.5 \mathrm{~cm}^{-1}$. Because all the phonon modes are blue shift with respect to those of the bulk values [3], the GaN epilayer is therefore under a compressive stress. The origin of the residual stress can be easily understood as follows. For the GaN film grown on sapphire, the lattice mismatch, $13.4 \%$, is quite large, which will result a residual stress in the epifilm. Nevertheless, the stress due to the lattice mismatch relaxes at a film thickness of less than several nanometres near the heterointerface during

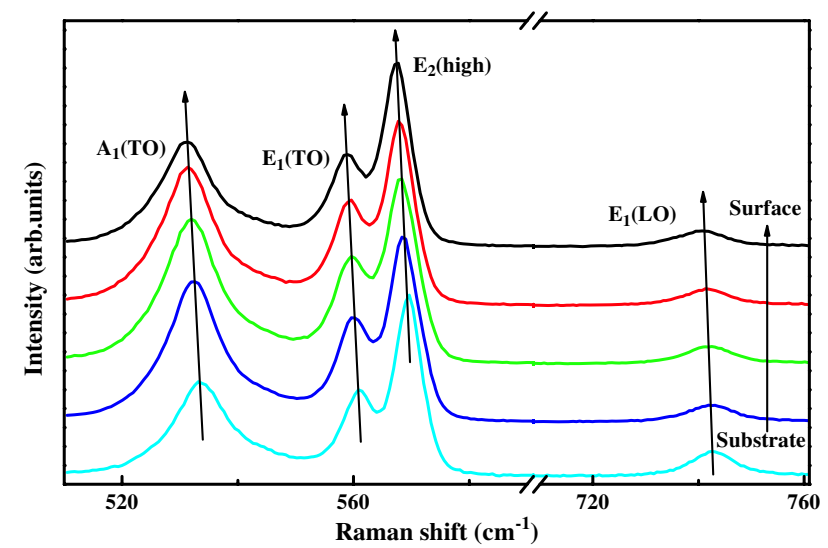

Figure 2. Cross-sectional Raman scattering spectra of the GaN epilayer taken at different distances away from the epifilm-substrate interface.

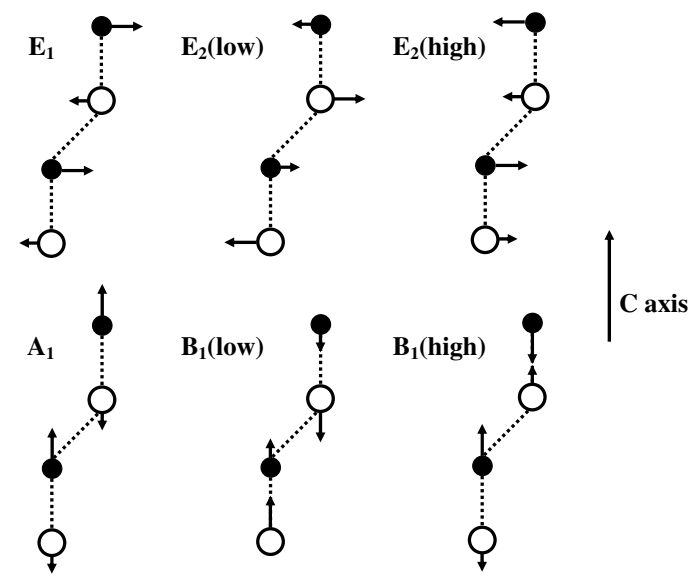

Figure 3. Atom vibration modes in hexagonal $\mathrm{GaN}$ with the $c$ axis upward.

growth [9]. Therefore, the residual compressive stress in the epilayer arises mainly from the large difference in the thermal expansion coefficients between $\mathrm{GaN}$ and sapphire when the sample was cooling down from the growth temperature.

To elucidate this intriguing property further, the Raman scattering spectra were performed with the laser light incident on the cross section instead of the surface. The polarized micro-Raman scattering spectra taken at various distances away from the epifilm-substrate interface are shown in figure 2. Four Raman active phonons-i.e., $\mathrm{A}_{1}(\mathrm{TO}), \mathrm{E}_{1}(\mathrm{TO})$, $E_{2}($ high $)$ and $E_{1}(L O)$ - are present in the spectra with the phonon peaks exhibiting a redshift with increasing distance away from the epifilm-substrate interface. It is known that the variation of Raman shift is an indication of the stress variation in the epilayer [10]. The redshift can be easily understood according to the configurations of phonon modes, as shown in figure 3, in which the $c$ axis points upward. For example, the $\mathrm{E}_{2}$ (high) mode corresponds to the atomic oscillation in the $c$ plane, and its mode frequency is therefore sensitive to the lattice strain in the $c$ plane [7]. When the biaxial compressive stress decreases, its phonon frequency also decreases. While for the $\mathrm{A}_{1}(\mathrm{TO})$ mode corresponding to the atomic oscillation 


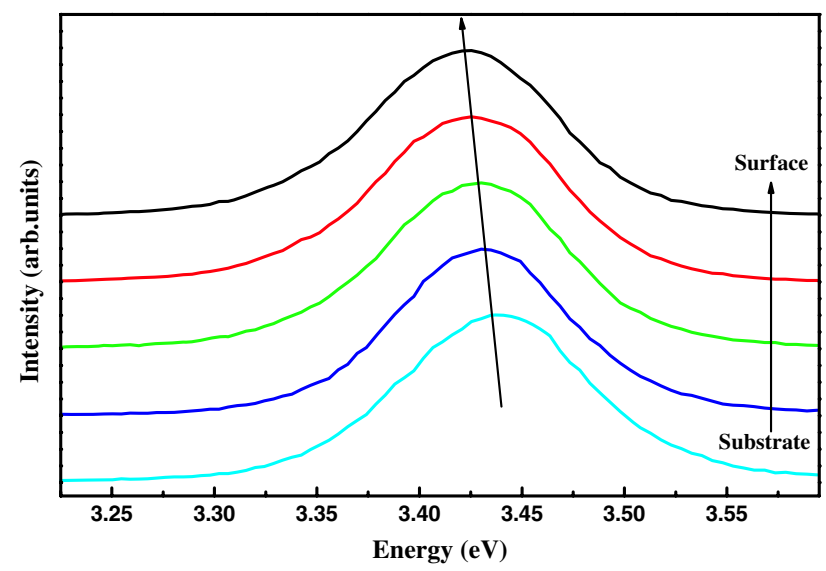

Figure 4. Cross-sectional cathodoluminescence spectra of the GaN epilayer taken at different distances away from the epifilm-substrate interface.

along the $c$ axis, it is not straightforward to understand the redshift when the biaxial compressive stress decreases. In the case of a biaxial strain, with the decreased compressive stress, an increase of the $a$-lattice constant accompanies a decrease of the $c$-lattice constant. Therefore, the fact that the $\mathrm{A}_{1}(\mathrm{TO})$ phonon peaks are redshifted in figure 2 seems to be counterintuitive to what might be expected when the compressive stress decreases. However, following Wagner et al [11], in the case of a biaxial strain, the bond length along the $c$ axis $\left(d_{/ /}=u c\right)$ increases, and the mode frequencies corresponding to the atomic oscillations along the $c$ axis, such as $A_{1}(T O)$ and $A_{1}(L O)$, exhibit a redshift when the compressive stress decreases due to the combined influence of phonon deformation potentials and elastic constants.

The observed variation of the residual strain along the growth direction in thick GaN films can be understood as follows. Because the thermal expansion coefficient of sapphire is larger than that of $\mathrm{GaN}$, the lattice of sapphire would contract more than that of $\mathrm{GaN}$ during cooling after growth. Therefore, the compressive stress exists in the GaN epilayer, and it would be the greatest near the epifilm-substrate interface between $\mathrm{GaN}$ and sapphire. The compressive stress will gradually relax away from the epifilm-substrate interface, and it results in a red shift of the Raman scattering spectra along the growth direction, as shown in figure 2. Furthermore, the description given in the previous report is also very useful to understand the behaviour. Due to the elastic relaxation close to the free edge, the epilayer bulges out and the strain is transferred from the film to the substrate. Following Jain et al [12], we know that both the horizontal and vertical lattice planes and curved in the film as well as in the substrate and the stress along the growth direction near the edge of the epilayer gradually decreases away from the film-substrate interface. It is worth noting that if the epilayer thickness is larger than critical thickness, the number of interface defects such as microcracks and/or dislocations increases abruptly [9]; it is expected that both of the plastic and elastic relaxations do occur in the epifilm and introduce anisotropy [13]

Let us now try to acquire additional evidence for the residual strain by performing the CL spectra with the electron beam scanned across the cross section, as shown in figure 4 .

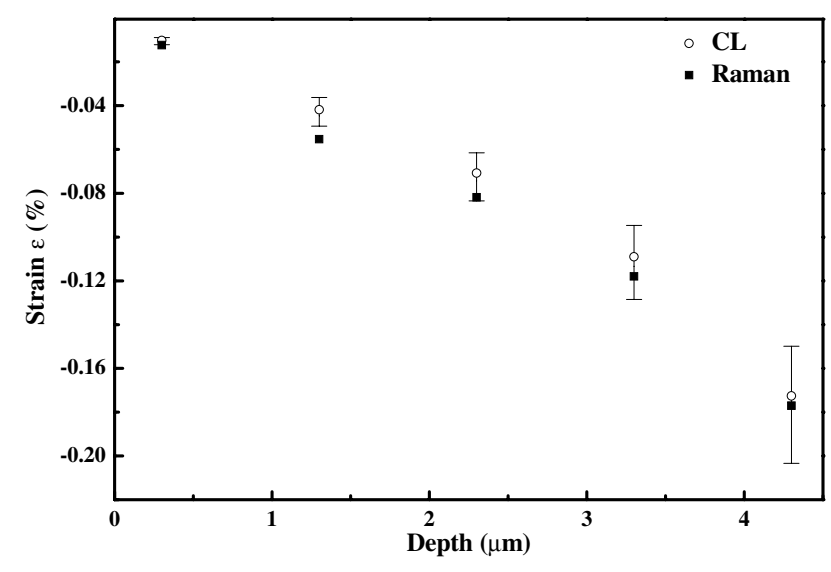

Figure 5. Variation of strain with depth of the GaN epifilm.

We can clearly see that the CL peak shows a red-shift from the epifilm-substrate interface towards the surface of the epilayer. It is well known that the energy band gap of a semiconductor can be significantly affected by stress. A tensile stress will cause a decrease of the energy band gap, while a compressive stress results in an increase. The redshift of the band gap therefore indicates that the residual compressive stress decreases with distance away from the epifilm-substrate interface, which is in good agreement with the Raman scattering measurement. Following Zhao et al [14], a biaxial stress of one GPa shifts the band gap by $21.1 \pm 3.2 \mathrm{meV}$ and the $\mathrm{E}_{2}$ (high) mode by $2.56 \mathrm{~cm}^{-1}$. In figure 2, the softening of the $\mathrm{E}_{2}$ (high) mode from the epifilmsubstrate interface to the surface of the epifilm is about $2 \mathrm{~cm}^{-1}$, which corresponds to a change of the band gap of about $16.5 \pm 2.5 \mathrm{meV}$. This value agrees very well with the peak shift of $16 \mathrm{meV}$ obtained from CL spectra, as shown in figure 4.

In order to have a roughly estimated magnitude of the residual stress along the growth direction, the $\mathrm{E}_{2}$ (high) frequency at the edge of the surface is chosen as the stressfree reference, because it is nearly mechanically unconstrained by neighbouring $\mathrm{GaN}$ and by the substrate [7, 12]. Indeed, our Raman scattering measurements indicate that the phonon frequency, $567.3 \mathrm{~cm}^{-1}$, at the edges of the surface has the smallest value. The residual thermal strain $\varepsilon$ along the growth direction can therefore be estimated according to the expression given by [15]

$$
\Delta \omega_{\lambda}=\left(2 a_{\lambda}(x)-\frac{2 C_{13}(x)}{C_{33}(x)} b_{\lambda}(x)\right) \varepsilon_{/ /},
$$

where $a_{\lambda}$ and $b_{\lambda}$ are phonon deformation potentials and $C_{13}$ and $C_{33}$ are elastic constants. For $\mathrm{GaN}$, the following values were used: $a_{\lambda}\left(E_{2}(\right.$ high $\left.)\right)=-850, b_{\lambda}\left(E_{2}(\right.$ high $\left.)\right)=-920$ in $\mathrm{cm}^{-1}$ [15], and $C_{13}\left(E_{2}(\right.$ high $\left.)\right)=106, C_{33}\left(E_{2}(\right.$ high $\left.)\right)=398$ in GPa [16]. To estimate the strain from CL spectra, the band gap of the stress-free value is taken as $3.42 \mathrm{eV}$ [3], and according to a previous report, a biaxial stress of one GPa shifts the band gap and the $\mathrm{E}_{2}$ (high) mode by $21.1 \pm 3.2 \mathrm{meV}$ and $2.56 \mathrm{~cm}^{-1}$, respectively [14]. The residual thermal strain $\varepsilon$ along the growth direction thus can be estimated both from Raman scattering and CL spectra, as shown in figure 5, in which negative $\varepsilon$ indicates that the strain is compressive. We 
can clearly see that even though the implemented techniques for both measurements are very different, the obtained results are in good agreement. Therefore, we have unambiguously demonstrated the existence of residual thermal stress in GaN epifilms.

Finally, it is worth noting that because the existence of strain arises from residual thermal stress, it should be possible to compare the measured strain with the estimated by $\varepsilon=\Delta \alpha \Delta T$, where $\Delta \alpha$ is the difference in the thermal expansion coefficients between $\mathrm{GaN}$ and sapphire and $\Delta T$ is the difference between the growth temperature and room temperature. Using $\Delta \alpha=2.05 \times 10^{-6}{ }^{\circ} \mathrm{C}^{-1}$ [9] and $\Delta T=1045^{\circ} \mathrm{C}$, the estimated strain is $2.14 \times 10^{-3}$, which is in good agreement with the strain of $1.87 \times 10^{-3}$ obtained by the Raman scattering spectrum. The slight difference between the estimated and measured strain can be ascribed to the enhancement of interface defects such as microcracks and/or dislocations [9].

\section{Conclusion}

In conclusion, based on cross-sectional Raman scattering and CL spectra, we firmly establish that there exists a compressive strain along the growth direction in $\mathrm{GaN}$ epifilms. The result shown here should be very important for the understanding and application of nitride semiconductors.

\section{Acknowledgments}

This work was supported by the National Science Council of the Republic of China.

\section{References}

[1] Mohammad S N, Salvador A A and Morkoc H 1995 Proc. IEEE $\mathbf{8 3} 1306$

[2] Nakamura S, Senoh M, Iwasa N and Nagahama S 1995 Japan. J. Appl. Phys. 34 L797

[3] Pankove J I and Moustakas T D (ed) 1998 Gallium Nitride (GaN) I, Semiconductors and Semimetals vol 50 (San Diego, CA: Academic)

[4] Rieger W, Metzger T, Angerer H, Dimitrov R, Ambacher O and Stutzmann M 1996 Appl. Phys. Lett. 68970

[5] Shan W, Fischer A J, Song J J, Bulman G E, Kong H S, Leonard M T, Perry W G, Bremser M D and Davis R F 1996 Appl. Phys. Lett. 69740

[6] Dadgar A, Poschenrieder M, Reiher A, Bläsing J, Christen J, Krtschil A, Finger T, Hempel T, Diez A and Krost A 2003 Appl. Phys. Lett. 8228

[7] Chen J W, Chen Y F, Lu H and Schaff W J 2005 Appl. Phys. Lett. 87041907

[8] Arguello C A, Rousseau D L and Porto S P S 1969 Phys. Rev. 1811351

[9] Hiramatsu K, Detchprohm T and Akasaki I 1993 Japan. J. Appl. Phys. 321528

[10] Perlin P, Jauberthie-Carillon C, Itie J P, Miguel A S, Grzegory I and Polian A 1992 Phys. Rev. B 4583

[11] Wagner J-M and Bechstedt F 2002 Phys. Rev. B 66115202

[12] Jain S C, Harker A H, Atkinson A and Pinardi K 1995 J. Appl. Phys. 781630

[13] Calle F, Alvarez A L, Sacedon A, Calleja E and Munoz E 1995 Phys. Status Solidi a 152201

[14] Zhao D G, Xu S J, Xie M H, Tong S Y and Yang H 2003 Appl. Phys. Lett. 83677

[15] Kontos A G, Raptis Y S, Pelekanos N T, Georgakilas A, Bellet-Amalric E and Jalabert D 2005 Phys. Rev. B 72155336

[16] Polian A, Grimsditch M and Grzegory I 1996 J. Appl. Phys. 793343 